\title{
Rigorous evaluation of a substance use and teen pregnancy prevention program for American Indian girls and their female caregivers: a study protocol for a randomized controlled trial
}

Rachel A. Chambers * (D), Jaime Begay, Hima Patel, Jennifer Richards, Danielle Nelson, Summer Rosenstock, Ronni Huskon, Kristin Mitchell, Tiffani Begay and Lauren Tingey

\begin{abstract}
Background: Early sexual initiation is associated with higher risk for sexually transmitted infection, teen pregnancy, domestic violence and substance use in later adolescence and early adulthood. Native American adolescents are more likely to have early sexual initiation compared to other racial/ethnic groups. Few programs designed with and for Native adolescents to delay sexual initiation and substance use have been tested through rigorous evaluations. This is the protocol for the randomized controlled trial of the Asdzáán Be'eena' program, a teen pregnancy and substance use prevention program for young Native girls and their female caregivers.

Methods: $N=410$ female adolescents ages 10-14 and their female caregivers will be enrolled in the study and randomized to the intervention or control arm. The intervention consists of the 11-session Asdzáán Be'eena' program. The control arm consists of mailed non-monetary incentives. All participants will complete evaluations at baseline and 3 follow-up timepoints (immediate, 6 and 12 months post intervention). Evaluations include measures to assess protective factors associated with delayed sexual initiation and substance use.

Discussion: This is one of the first rigorous evaluations of a gender-specific, culturally tailored teen pregnancy and substance use primary prevention program for Native girls and their female caregivers. If proven efficacious, Native communities will have a culturally appropriate program for promoting protective factors associated with delayed substance use and sexual risk taking.
\end{abstract}

Trial registration: NCT04863729; April 27, 2021.

Keywords: Primary prevention, Intergenerational, Teen pregnancy prevention, Substance use prevention, Female, Native American, Culture

\footnotetext{
* Correspondence: rstrom3@jhu.edu

Johns Hopkins Center for American Indian Health, Johns Hopkins Bloomberg School of Public Health, 415 North Washington Street, Baltimore, MD 21231, USA
}

C C The Author(s). 2021 Open Access This article is licensed under a Creative Commons Attribution 4.0 International License, which permits use, sharing, adaptation, distribution and reproduction in any medium or format, as long as you give appropriate credit to the original author(s) and the source, provide a link to the Creative Commons licence, and indicate if changes were made. The images or other third party material in this article are included in the article's Creative Commons licence, unless indicated otherwise in a credit line to the material. If material is not included in the article's Creative Commons licence and your intended use is not permitted by statutory regulation or exceeds the permitted use, you will need to obtain permission directly from the copyright holder. To view a copy of this licence, visit http://creativecommons.org/licenses/by/4.0/. The Creative Commons Public Domain Dedication waiver (http://creativecommons.org/publicdomain/zero/1.0/) applies to the data made available in this article, unless otherwise stated in a credit line to the data. 


\section{Introduction}

\section{Background and rationale}

Extensive research documents how both early sexual debut and substance use intiation adversely impact the health of an individual. Specifically, early sexual debut increases the likelihood of poor sexual and reproductive health outcomes, such as sexually transmitted infections (STI) and unintended teen pregnancy [1-4]. It is also associated with a greater number of sexual partners and increased risk of being a victim of intimate partner violence [4-6]. In terms of substance use, early initiation of substance use adversely impacts the cognitive and emotional growth and development of adolecents [7-9].

Early sexual debut and substance use are also costly for the individual, society and tax payer. A recent analysis conducted by Rotz et al. found large economic savings of preventing teen sexual activity and substance use. The analysis estimates a net benefit of up to $\$ 52,109$ for females and $\$ 27,861$ for males from delaying voluntary sexual activity to age 15 or older [10]. For substance use, the estimated cost savings for preventing underage drinking is estimated to be up to $\$ 12,313$ for individuals [10].

Not surprisingly, substance use initiation is one of the most significant risk factors for sexual initiation and unprotected sex among adolescents. In fact, these behaviors tend to co-occur, particularly among more vulnerable subgroups of adolescents [11, 12]. These behaviors also share similar risk factors including lack of family cohesion [13-17] and poor adolescent functioning (having high levels of internalizing and externalizing behaviors). They also share many protective factors including connection to culture [18-21], parent-child relationship, self-efficacy and future aspirations [21-26]. Programs targeting these shared risk and protective factors may have a dual impact: reducing both sexual risk taking and substance use among adolescents.

National data show Native American (Native) adolescents are more likely to initiate sex before age 13 than all other U.S. racial/ethnic groups, except African American adolescents [27]. Native high school youth are also more likely to have multiple sex partners and to ever have had sex compared to youth of all races [27, 28]. Native youth also experience higher rates of STIs and teen pregnancy than their non-Native counterparts; in 2018, U.S. national Chlamydia and Gonohrrea rates among Native Americans were 3.7 and 4.6 times higher than that among Whites [29]. Further, in 2017, the national teen birth rate among 15-19 year old Native Americans was the highest of all races and ethnicities [30, 31]. It follows that Native adolescents have higher rate of substance use and abuse. Compared to all U.S. adolescents, in 2018, Native adolescents ages $12-17$ are more likely to engage in past-month binge drinking (5.7\% vs. $4.9 \%)$, marijuana use (11.1\% vs. $7.4 \%)$, and other illicit drug use (12.8\% vs. $8.7 \%)$ [32].
Given the adverse outcomes of early sexual activity and substance use and the high rates of sexual risk taking and substance use among Native adolescents, prevention efforts to delay these behaviors in Native communities are warranted [33]. These efforts may be more advantageous if delivered during early adolescence (ages 10-14), before a child initiates sex or substance use. Early adolescence is a key developmental period in which children continue to solidify their values and relationships, and learn foundational life skills [34-37]. Hindered growth in these areas during this time period has been linked to poorer health outcomes including early sexual initiation, substance use and abuse in later adolescence and adulthood [33]. Thus, programs that promote values, focus on building healthy relationships and teach life skills such as problem-solving and goal-setting in early adolescence are needed.

As opposed to many Western societies, most Native cultures do not have a self-centered orientation to health, but instead have family- and community-oriented health frameworks [38]. Thus, intergenerational programming may be more culturally appropriate [39] than individuallevel programs. In addition to cultural congruency, engaging parents alongside adolescents in programming may be advantageous as they can support and reinforce behaviors $[40,41]$. While robust efforts have begun to address behavioral health disparities among Native adolescents, currently there are no intergenerational, efficacious primary prevention programs targeting the dual threat of sexual risk taking and substance use for young Native adolesents [42-44]. This study seeks to fill this gap.

\section{The current study}

The objective of the is to assess the efficacy of a program called Asdzáán Be'eena' (Female Pathways, AB) for increasing protective factors associated with delayed substance use and sexual risk taking among Native girls in early adolescence (ages 10-14) through a randomized controlled trial (RCT). This paper describes the study protocol.

\section{Objectives}

The study will test the efficacy of the AB program on risk and protective factors associated with delaying adolescent substance use and sexual initiation, as well as actual sexual initiation and substance use. By assessing protective and risk factors as well as behaviors among the adolescent and the caregiver, the study team will be able to assess how each of these are impacted by the intervention, and how these interact with one another to moderate program impact.

Primary research questions include: 1) Is the intervention effective in increasing adolescents' intention to abstain from sex (intention to abstain was chosen as opposed to sexual initiation as we do not anticipate high 
rates of sexual initiation for the duration of the followup time period); 2) Is the intervention effective in improving caregiver-adolescent relationships. Secondary research questions include: 1) Is the intervention effective in improving adolescent risk and protective factors associated with delayed sexual initiation and/or substance use initiation; 2) Is the intervention effective in improving caregiver risk and protective factors associated with delayed adolescent sexual initiation and/or adolescent substance use initiation; 3) Is the intervention effective in delaying sexual initiation and substance use initiation among adolescents; 4) Is the intervention effective in reducing substance use among enrolled caregivers.

The study team hypothesizes a higher proportion of adolescents in the intervention group will state they intend to abstain from sex in the next year and until they graduate high school compared to the control group at 6and 12-months post intervention. Additionally, we hypothesize that there will be 1) an increase in caregiveradolescent communication at 6- and 12-months post intervention as measured by a communication scale adapted from a trial with Ojibwe adolescents, 2) an increase in caregiver-adolescent quality time together at 6and 12-months post intervention as measured by a quality time with parent scale adapted from a trial with Ojibwe adolescents, 3) an increase in maternal warmth at 6- and 12-months post intervention as measured by the Authoritative Parenting Index, and 4) an increase in caregiver monitoring at 6- and 12-months post intervention as measured by the parental monitoring scale.

\section{Trial design}

The study is a two-armed randomized controlled trial examining the primary outcomes of adolescent-caregiver relationship, adolescent intention to have sex and adolescent adaptive/maladaptive functioning at 12 months post intervention. The trial is being conducted at two study sites on the Navajo Nation with Navajo girls ages 10-14 and their female caregiver. Randomization will be performed with a stratified block randomization sequence to ensure a 1:1 allocation of study conditions within each of the two study sites Fig. 1.

\section{Methods/design}

\section{Study setting}

The study will be conducted by the Navajo Nation in partnership with Johns Hopkins Center for American Indian Health (Center). The study design was approved by the appropriate Navajo governing bodies including Chapters, Agency Councils and Health Boards as well as the JHU research review board and the Navajo Nation Human Subjects Research Review Board (NNHRRB). This manuscript was approved by the NNHRRB. All deviations from the protocol will be approved by IRBs before being implemented. Once approved, they will be updated in clinicaltrials.gov by the PI. As needed, participants will be reconsenting utilizing consenting documents that include changes to the protocol and have been approved by the appropriate IRBs.

The study is community based intervention and conducted on the Navajo Nation in the United States of America.

\section{Study staffing}

This study is being conducted at two study sites on the Navajo Nation. The Center has had a physical office and has conducted projects in partnership with the Navajo Nation for over 30 years. Each site has two family health coaches (FHCs), one independent evaluator (IE) and one site coordinator. Other members of the study team, including the principal investigator, program manager, program coordinator and evaluator, are located at the Center's administrative office in Baltimore, Maryland. The field manager is located in Arizona near the Navajo Nation. All members of the study team are full-time Johns Hopkins employees and are thoroughly trained in human subjects research prior to working with participants. Members of the Baltimore team and the field manager make frequent trips (at least bi-annual) trips to the study sites to conduct training and quality assurance.

\section{Family health coaches}

FHCs are Navajo women living in one of the study communities. All speak some Navajo. All FHCs will complete extensive $(>60 \mathrm{~h})$ of training in the program curriculum as well as skills necessary to deliver the program including home-visiting, group facilitation, and boundary setting. They will be responsible for delivering the $A B$ program to dyads. They will also be responsible for delivering the non-monetary retention gifts to dyads in the control group.

\section{Independent evaluators}

Independent Evaluators are Navajo women living in one of the two study communities. All speak some Navajo. All IEs will complete extensive training $(>40 \mathrm{~h})$ in the study protocol including recruitment, consenting and data collection. IEs will recruit participants, administer informed consent, receive parental permission and adolescent assent. IEs will also administer all assessments and provide gift cards for assessment completion.

\section{Site coordinators}

Site Coordinators are Navajo women living in one of the two study communities. They will provide on-site support to all local study staff. 


\section{Participants and eligibility criteria}

Participants are Native female adolescents and their female caregiver (referred to as dyads). Inclusion criteria for the adolescent includes: Native American ethnicity, 10-14 years of age, living within 50 miles of one of the two study sites, and written parental permission and adolescent assent. Participant inclusion criteria for the caregiver includes: Native American ethnicity, $\geq 18$ years of age, caregiver to a female child age 10-14 years who will enroll in the study with them, living within 50 miles of a study site, and written informed consent. If the caregiver is not the legal guardian of the adolescent, the adolescent's legal guardian must agree for the caregiver to participate. To honor cultural views around caregiving, female caregivers who live outside of the home but play a pivotal role in child rearing are welcome to participate. To enroll, both members of the dyad (adolescent and caregiver) must be eligible and enroll. Adolescents in foster care are ineligible to participate.

\section{Interventions}

\section{The Asdzaan Be'eena' program}

The $A B$ program is described in detail elsewhere (Chambers 2021). A short description is included here.

\section{Program development}

Program development occurred from 2016 to 2018 and included the following: 1) 12 focus groups and 15 interviews with Navajo girls ages $8-25$, mothers and grandmothers, fathers and grandfathers, as well as traditional practitioners, 2) 10 Community Advisory Board (CAB) meetings each consisting of 8-12 community leaders, 3 ) administration of a survey to 400 Navajo adult women to assess preferences and experiences with puberty, 4) a three-day intensive workshop with cultural specialists and 5) an extensive review of the literature to identify other programs and requisite risk and protective factors to delay sexual initiation and substance use among $\mathrm{Na}-$ tive girls in pre- and early adolescence. Lessons were drafted by the Johns Hopkins curriculum team in consultation with cultural experts, reviewed by the $C A B$ and piloted with $N=47$ dyads. Evaluation assessments were piloted with participating mothers and daughters at baseline and 3 month time points. Pilot findings indicate the program positively impacted communication between girls and their female caregivers $(p=0.2 ; p<.001)$, improved cultural $(p=.02)$ and reproductive health knowledge $(p=.001)$, while simultaneously reducing internalizing behaviors $(p=.001)$, deviant disorder symptoms $(p=.04)$, and attention deficit disorder $(p=.03)$ at 3 months post program completion. Female adolescents who completed the program were also more likely to state that they planned to wait to have sex until married ( $25 \%$ vs. $70 \%, p=.218$ ). This preliminary data justified rigorous evaluation of the $A B$ program for impacts on delayed sexual and substance use initiation.

\section{Program curriculum}

The $A B$ program is a culturally grounded curriculum consisting of eleven lessons organized according to the teachings of the Navajo creation story, designed specifically for Navajo females. Lessons also provide knowledge and skills necessary for delaying substance use and sexual initiation as identified through the focus groups, indepth interviews and literature review. All lessons are taught by a FHC, and consist of three to five activities designed to teach a skill or provide knowledge about a topic (see table below). At the end of each lesson, 1) the FHC reviews key teachings from the lesson, 2) dyads practice three to five Navajo vocabulary words, 3) the FHC provides the dyad with a positive affirmation, 4) the dyads are given worksheets with information and activities completed during the session, and 5) the FHC gives a scheduling reminder for the next lesson.

\section{Program implementation}

The $\mathrm{AB}$ curriculum is implemented through a mix of individual dyad and group formats (see Table 1). Individual dyad lessons are taught by one FHC to a dyad in their home or another place of their choosing (e.g. the local program office, a local school or clinic). Snacks are provided to dyads during these sessions. Group lessons are taught by 2 FHCs (facilitator and co-facilitator) to groups of 7-12 dyads in a central location in the community (e.g. chapter house, local clinic or school). Meals are provided to dyads during group sessions and transportation to group sessions is provided as needed. Participants complete the program in cohorts of 7-12 dyads. All members of a cohort participate in group lessons together.

\section{Control condition}

The control condition consists of the delivery of four non-monetary retention items totaling $<\$ 20 /$ dyad to the participant's home. Retention items were selected by the community and provided as a way to continue to stay in contact with families for follow-up data collection.

\section{Discontinuing or modifying intervention}

Participants will not be allowed to change groups. They will be able to stop participating at the study at any point. Study staff are trained to identify and report adverse events. In the event of an adverse event, the study staff member will call the PI and follow-up with an email detailing the event. All adverse events will be sent to the IRB according to their required schedule. The study will be reviewed by approving IRBs annually. There is no 
Table 1 AB Lesson Delivery Format and Content

\begin{tabular}{lll}
\hline Lesson number & Format & Topic \\
\hline 1 & Individual dyad & $\begin{array}{l}\text { Program introduction; discussion of the meaning of the clan system; importance of self-reflection in } \\
\text { Navajo culture }\end{array}$ \\
3 & Individual dyad & $\begin{array}{l}\text { Identifying role models; respect for self, others and mother earth } \\
\text { Building the caregiver adolescent relationship; strategies for improved communication }\end{array}$ \\
4 & Group & Developing support networks; Navajo introductions; family roles and values \\
5 & Individual dyad & Navajo history, Navajo teachings related to puberty; Introduction to the female reproductive system \\
6 & Group & Thinking positively; problem solving skills development \\
8 & Individual dyad & Communication styles; dealing with peer pressure; refusal skills \\
9 & Individual dyad & Community and family values; support networks; Kinaaldá (Navajo coming of age ceremony) teachings \\
& Group & $\begin{array}{l}\text { Reproductive Health 101: puberty and pregnancy; healthy hygiene during puberty; skills to improve } \\
\text { caregiver-adolescent communication about sensitive topics }\end{array}$ \\
10 & Caregiver only: safe sex practices \\
11 & Individual dyad & Self-esteem; identifying personal strengths; goal setting skills \\
\hline
\end{tabular}

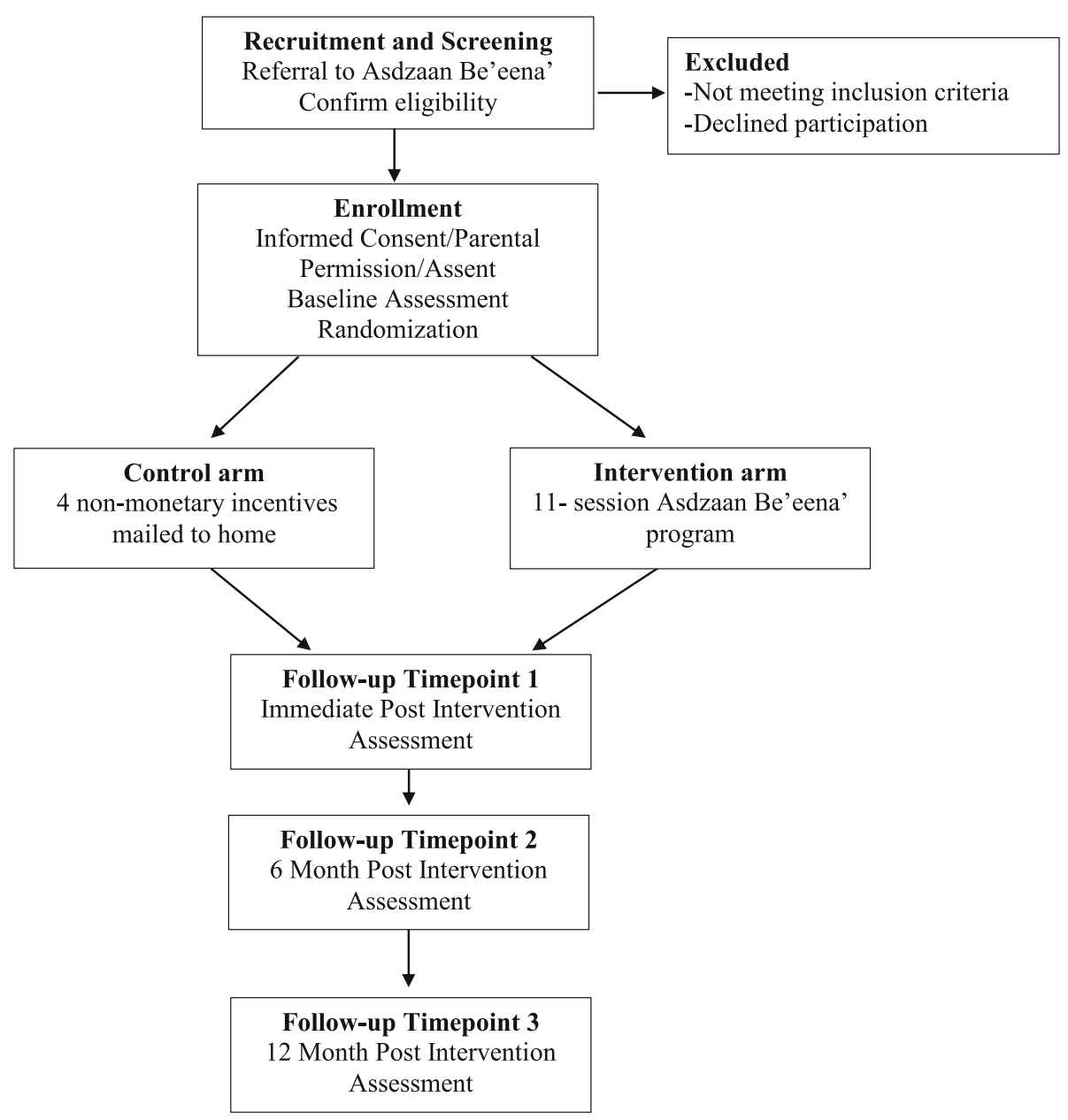

Fig. 1 Trial Design 
post-trial care or compensation for those who suffer harm from trial participation.

\section{Recruitment and consent}

Participants will be recruited through a combination of non-probability and snowball sampling. First, recruitment fliers will be posted in community gathering spots (i.e., supermarket, community centers, health and human services offices, fitness centers, etc.), on social media (e.g. Facebook) and disseminated during public gatherings through informational booths. Second, caregivers enrolled in the project will be asked to refer other caregivers who may be eligible and interested in the program. Third, IEs will provide information about the study to local agencies that provide services to eligible participants (e.g. Office of Self Reliance, local schools, counseling services etc.) and provide them with referral forms. Independent Evaluators will collect referral forms from these agencies on a regular basis (e.g. weekly). All recruitment materials have contact information for the local project coordinator. To learn about the project and enroll, interested individuals can call the local project coordinator. A study staff member will also reach out to referred caregivers or parents of a referred child. When contact is made with a potential participant, the IE will review a brief script describing the study and assess for eligibility. For those individuals meeting eligibility criteria who express interest in study participation, the study staff member will work with them to set up a time to complete informed consent/parental permission/ assent and work with the participant to contact the caregiver who will enroll with them (adolescent and adolescent's female caregiver). The informed consent process will include a review of how personal information will be collected, shared and maintained to protect confidentiality of the participant's study data. After both the caregiver and adolescent participants have completed informed consent and adolescent assent respectively, they will complete the baseline assessment and schedule their first lesson.

\section{Retention}

Participants will receive gift cards for participation in assessments (\$20 per assessment time point per participant). Additionally, FHCs and IEs will do all they can to keep participants enrolled and engaged including providing transportation to study visits and snacks or meals at these visits. Participants will only be dropped from the program if they request. Outcome data will be collected from all participants regardless of their intervention completion status.

\section{Outcomes}

Assessments will be administered at baseline, immediately following program completion and 6- and 12- months following program completion (see Table 2). Data will be collected in participants' homes or another private location via a tablet using Research Electronic Data Capture (REDCap). Caregivers will complete all assessments via self-report. Adolescents will complete portions of the outcome assessment asking about sexual and substance use behaviors via self-report. All other portions will be be completed via interview. Participants will be given $\$ 20$ after completion of the baseline, immediate, six and twelve month post intervention assessments. All measure items were previously piloted with adolescent and caregivers from the community to assess comprehension, language, and cultural relevance. Edits were made accordingly and pilot participants were not eligible for the RCT.

Please see Table 2 for a description of the outcome measures, including method and frequency of administration.

\section{Data management}

All data will be directly entered into REDCap by an intervieweer (IE) or the participant. If assessments are completed via paper, they will be entered by the IE. A data manager will review all data entered into REDCap to ensure completeness and conduct data quality assurance (including checking ranges for variables as applicable). All data will be stored via REDCap, a secure, HIPPA compliant, data collection and storage database. Data will be analyzed part way through the trial by the evaluator. The study management team (including the evaluator, the PI and the program manager) will review data and assess the need to continue the trial.

\section{Randomization and sample size}

This study will use a 1:1 randomized controlled trial design; the unit of randomization will be the individual dyad. Unique participant identification numbers will be used to randomize individual dyads to one of two study groups: intervention or control. Stratified block randomization, using random block size will be used to ensure a 1:1 allocation of study conditions across the two study sites. The sequence will be generated by the program evaluation via STATA and will not be shared with study staff who enroll participants.. Independent Evaluators will enroll participants and reveal study group after the participants complete the baseline assessment. The study assignments are sequentially numbers in sealed envelopes that are only opened at the time of randomization. No blinding will occur.

Sample size and statistical power estimates are predicated on 2 primary hypotheses: 1 ) that those randomized to the $\mathrm{AB}$ intervention will have improved parentadolescent communication compared to the control group, and 2), that those randomized to the $A B$ 


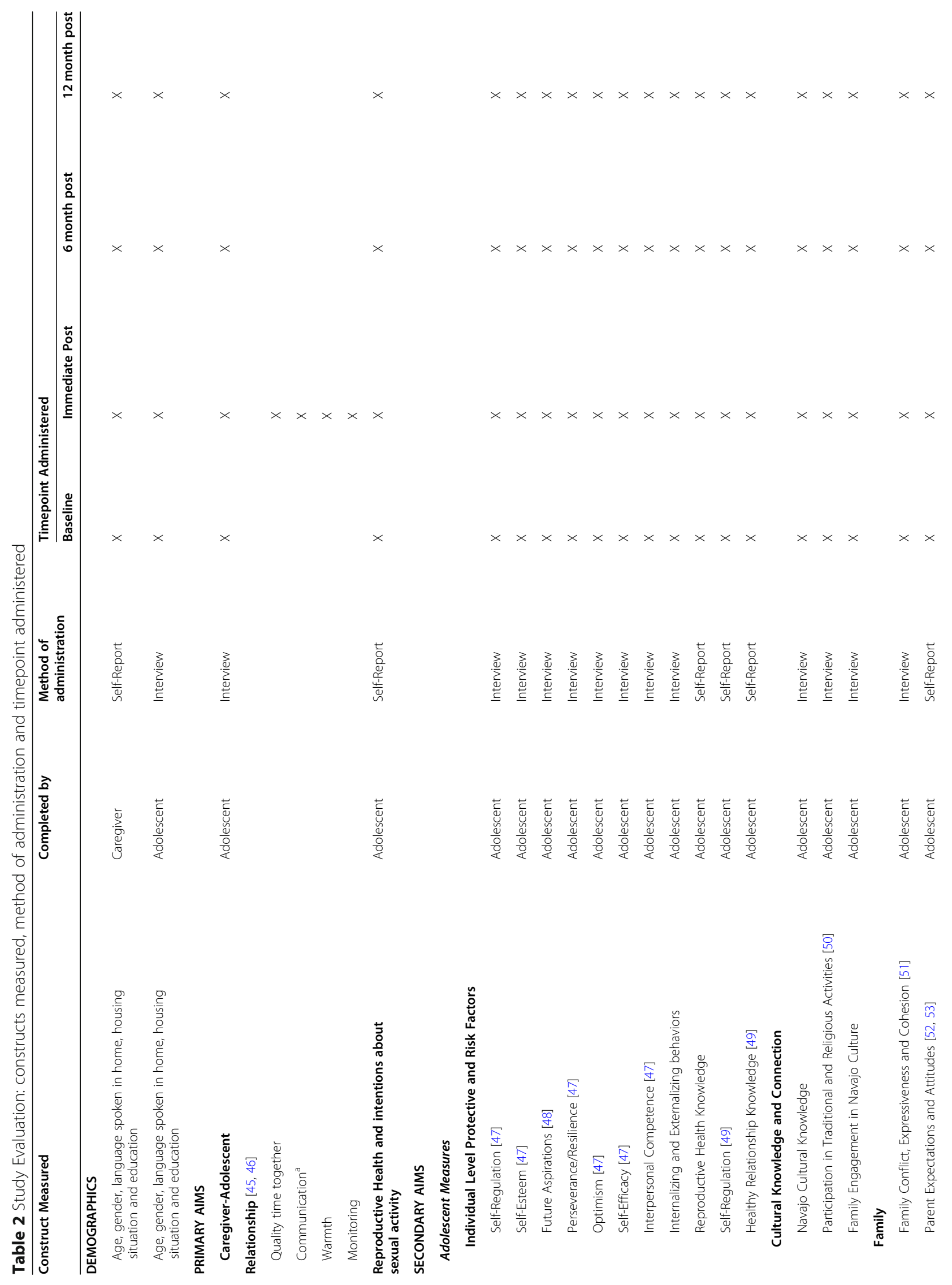




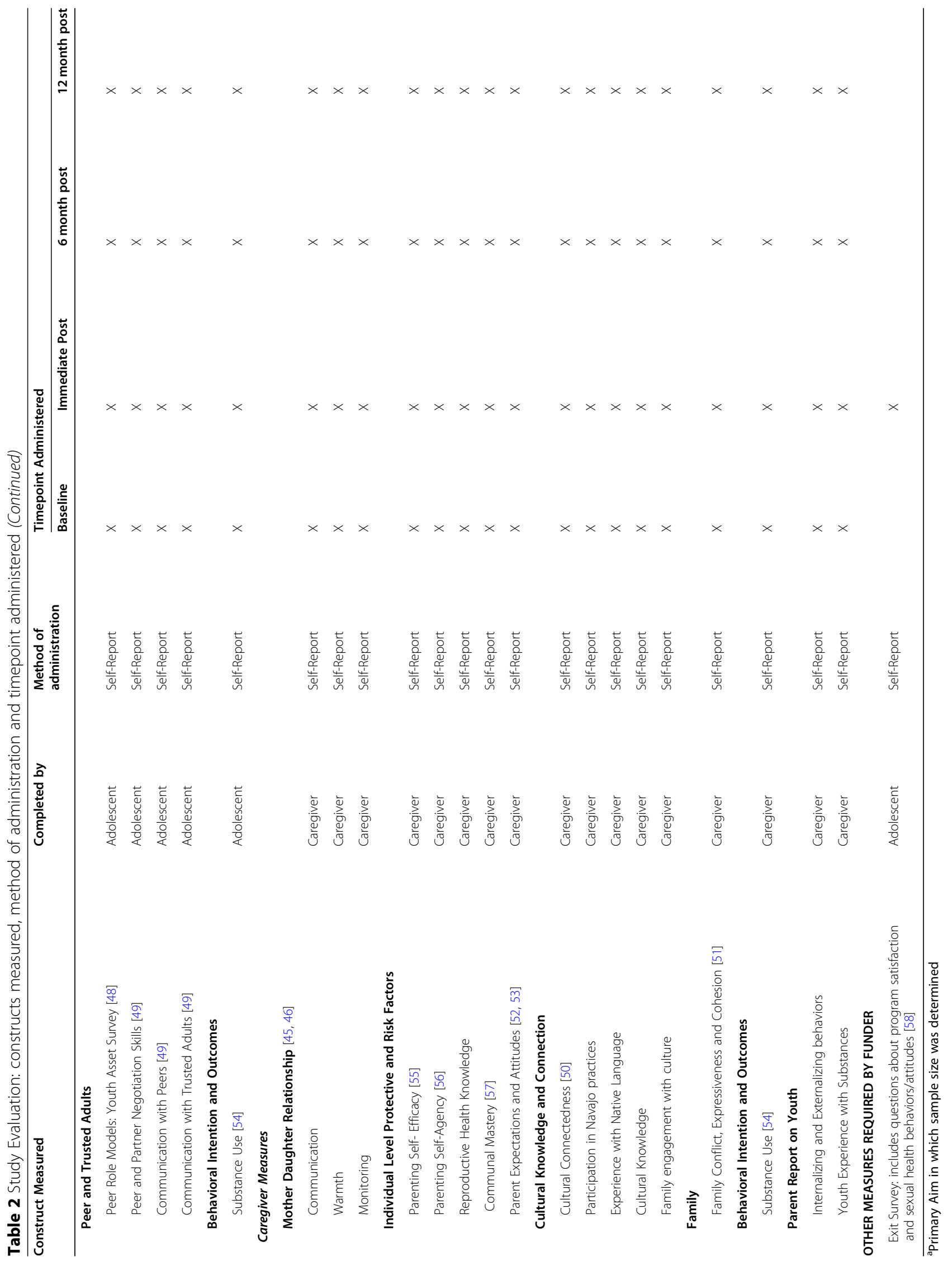


intervention group will have a higher proportion of participants who report they intend to abstain from sex.

The sample size of 410 dyads ( $n=205$ per study arm) will be sufficient to detect a 0.3 difference between study groups, with $90 \%$ power and 0.05 significance level, on the communication scale at 12 months post-intervention (estimating a mean score (SD) of 2.81(0.85) in the control group). This same sample size will be sufficient to detect an $18 \%$ between-group difference, with $80 \%$ power and 0.05 significance level, in the proportion of participants who report intention to abstain from sex at 12 months post-intervention (estimating $50 \%$ of the control group to report intention to abstain from sex at 12 months post-intervention). This sample size will also be able to detect meaningful differences in other key confirmatory research question outcomes at 12 months post-intervention. The following presents minimum detectable differences between the intervention and control groups with at least $80 \%$ power, given the assumptions listed above: 0.39 between-group difference in internalizing behaviors (estimating a mean score (SD) of 5.23 (0.99) in the control group), and 0.25 between-group difference in externalizing behaviors (estimating a mean score (SD) of 2.04 (0.63) in the control group). All estimates assume $10 \%$ attrition between enrollment and program completion and a $25 \%$ attrition rate between program completion and the 12 month follow-up time point.

\section{Statistical analysis}

The between study group equivalence of participant characteristics and outcome measures at baseline will be compared using Chi-squared tests (dichotomous/categorical data) and t-tests (continuous data). Study hypotheses related to the confirmatory questions will be initially tested under an "intent-to-treat" model in which data are analyzed according to treatment assignment at randomization. We will then conduct "completer analyses" on those subjects completing at least two-thirds of intervention sessions. Intervention impact will be evaluated by comparing primary study outcomes between intervention and control groups across the three time points: baseline, 6 months and 12 months postintervention. Initially, summary scores of outcomes will be stratified by participation in the intervention versus control groups and by time point, and will be compared using chi-square tests (binary or categorical outcomes), $\mathrm{t}$-tests and analysis of variance (continuous outcomes). Generalized linear mixed effects models (GLMM) accounting for within-group correlation across longitudinal data will then be applied. Outcomes will be modeled as a function of group assignment and time since intervention. The association between outcomes and time will be explored visually using scatterplots and lowess smoothers. Restricted cubic splines will be added to reflect deviations from linearity.

If siblings enter the study, they will be randomized to the same intervention group to limit contamination. In this case, analyses will be adjusted to take into account intra-sibling correlation as well. Additionally, sensitivity analyses will be conducted including and excluding siblings to examine the impact on study results. Missing data will be handled as follows: 1) identify erroneous data, 2) document reason(s) for missing and erroneous data to inform model development; 3) compare demographic characteristics of those who dropped out and those who did not to compare if data is missing at random; and 3) conduct sensitivity analysis to compare inferences that are based on different plausible reasons for missingness. Navajo and Hopkins investigators will collaborate with community partners in data interpretation to assure accuracy, cultural acceptance and relevance.

\section{Discussion}

This study protocol presents one of the first RCTs of a program designed by and for a Native community to promote protective factors associated with delayed substance use and sexual initiation among young girls.

This study has many strengths. First, as previously noted, $\mathrm{AB}$ was designed in consultation with Navajo cultural specialists and was shown to be culturally appropriate and acceptable in the pilot trial (Chambers 2021). Public health interventions with roots deriving from cultural teachings have brought inclusion and participant connection with the lesson content, thus solidifying the importance of integrating culture as the foundation for prevention programs specific to AI communities. Further, the program is designed specifically for female adolescents and draws on the traditional matrilineal society of the Navajo. Across ethnicities, female adolescents have distinct patterns and processes for underage substance use and sexual risk-taking that are different from male adolescents $[59,60]$. Some of these differences include females being more likely than males to use substances due to low self-esteem [61,62], and to be offered substances in private settings by female relatives [63, 64]. Also, lack of family support is a stronger risk factor for substance use and sexual initiation among Native female adolecsents than male adolescents [62]. Thus, a gender specific approach is a strength of this program.

Second, there is substantial evidence that parental involvement in teen pregnancy and substance use prevention programs is advantageous [65-67]. However, researchers have cited the lack of parental involvement in sexual health promotion programming as a gap in efforts to prevent teen pregnancy. They conclude that by providing the parent and adolescent the same health promotion and sexual risk prevention information, the 
parent is informed of what the adolescent is learning and can reinforce these teachings at home [68]. Given this, inclusion of a caregiver in this program alongside the adolescent is a major strength and may enhance and help to sustain positive program impact long after the completion of the program. Next, this study will assess the impact of the program on individual-level factors of both the caregiver and adolescent. This will not only allow for the understanding of program impacts on both caregiver and child, but will also allow the study team to better understand, 1) how adolescent and caregiver outcomes and risk/protective factors are associated with each other, and 2) how changes in adolescent and/or caregiver outcomes mediate change in outcomes in the other member of the dyad.

Third, the study builds on more recent research highlighting the importance of primary prevention programs delivered during early adolescence, when adolescents are less likely to have initiated risk-taking behaviors such as substance use and sex $[68,69]$. In addition, the $A B$ program is designed to simultaneously impact cooccurring risk behaviors that impact Native adolescents and communities at disproportionate rates. If $\mathrm{AB}$ is efficacious at reducing both sets of risk behaviors, it will fill a gap in the literature calling for evidence based interventions (EBIs) that work across domains and are not specific to one risk behavior (e.g. risks for substance use or early sex). Programs such as AB may be particularly beneficial for Native and other under-resourced populations for whom few comprehensive EBIs exist.

This study also has limitations. First, all outcomes are measured via self-report and/or via interview. While self-report is the most widely used methodology to assess behavior change, it is not without biases. There are also potential biases in interview administration, such as... [70]. However, steps have been taken to reduce potential biases. The IEs are highly trained in evaluation administration and human subjects research and will not deliver any part of either $A B$ program or condition nonmonetary gifts, limiting potential evaluator bias. Other methods to reduce bias include: 1) all IEs will be Native women from the local community, 2) the same IE will conduct the baseline and all follow-up assessments for each dyad, 3) all interviews will be conducted in person (vs. some in person and some via telephone/video call), and 4) the most sensitive questions focused on actual behavior and behavioral intention are conducted via selfreport (not interview).

Another limitation is that the follow-up time period is only 1 year. Since adolescents are 10-14 years of age when enrolling in this program, we do not expect a large proportion of adolescents to initiate substances or have sex by the 12 month follow-up timepoint. Thus, we do not expect to have adequate power to assess impact of $\mathrm{AB}$ on actual behaviors. However, we will have power to assess the impact of $\mathrm{AB}$ on behavioral intention. Studies indicate there is a strong, positive association between intention to abstain from sex and delayed sexual initiation [71]. Further, we will assess key protective factors that are associated with delayed sexual initiation and substance use among Native and non-Native adolescents [19, 23, 71].

Third, there is potential for some contamination between control and intervention groups, as this study will be conducted in a small communities where many families know each other. To limit contamination, we will individually randomize dyads and assign siblings to the same randomization group.

To our knowledge, this is the first RCT of a culturally grounded primary prevention program for teen pregnancy and substance use among female Native adolescents and their female caregivers. If $A B$ proves efficacious, there will be a program for Native families that promotes protective factors associated with delayed substance use and sexual risk taking. Additionally, we may deepen our understanding of the relationship between adolescent and caregiver individual and family level factors, how each of these contribute to risk taking behaviors among Native adolescents, and how shifts in adolescent individual level factors influence caregiver outcomes and vice versa.

\section{Abbreviations \\ Al: American Indian; AB: Asdzáán Be'eena'(Female Pathways); NNHR RB: Navajo Nation Human Subjects Research Review Board; Center: Center for American Indian Heallth; Native: Native American; RCT: Randomized Control Trial; FHCs: Family health coaches; IE: Independent evaluator; CAB: Community Advisory Board; GLMM: Generalized linear mixed effects models; EBls: Evidence-based interventions}

\section{Acknowledgements}

We would like to acknowledge the families who participated in this research and the tribal community partners who provided guidance and support for this work. We are forever grateful for their dedication to improving the health of families in their communities and around the work and for their invaluable leadership and guidance.

\section{Authors' contributions}

$R C, L T, J B$ and HP conceptualized the study design. RC and JB drafted the manuscript. All authors reviewed and approved the manuscript.

\section{Author's information}

All authors are members of a Center that has a 35 year history of working with American Indian communities in the Southwestern United States.

\section{Funding}

The author(s) disclosed receipt of the following financial support for the research, authorship, and or publication of this article: funding was received from the Administration for Children and Families, Families and Youth Services Bureau (\#90SR0087-01-00).

Availability of data and materials

Not Applicable. Only Study Co-Investigators have access to the final trial dataset. Trial results will be provided to community advisory boards and the Navajo Nation IRB through a report and presentation. The data set is owned by the Navajo Nation and will not be publicly available. 


\section{Declarations}

\section{Ethics approval and consent to participate}

This study was approved by the Johns Hopkins Bloomberg School of Public Health Institutional Review Board (IRB\#1436) and the Navajo Nation Human Subjects Research Review Board (NNR-15.223). All participants completed written informed consent (adult participants) or written parental permission and written assent (minor participants) prior to participating in any study activities.

\section{Consent for publication}

Not Applicable.

\section{Competing interests}

The authors declare that they have no competing interests.

Received: 5 May 2021 Accepted: 24 May 2021

Published online: 21 June 2021

\section{References}

1. Sandfort TGM, Orr M, Hirsch JS, Santelli J. Long-term health correlates of timing of sexual debut: results from a national US study. Am J Public Health. 2008;98(1):155-61. https://doi.org/10.2105/AJPH.2006.097444.

2. O'Donnell $L, O$ 'Donnell $C R$, Stueve A. Early sexual initiation and subsequent sex-related risks among urban minority youth: the reach for health study. Fam Plan Perspect. 2001;33(6):268-75. https://doi.org/10.2307/3030194.

3. Kaestle CE. Young age at first sexual intercourse and sexually transmitted infections in adolescents and young adults. Am J Epidemiol. 2005;161(8): 774-80. https://doi.org/10.1093/aje/kwi095.

4. Coker AL, Richter DL, Valois RF, McKeown RE, Garrison CZ, Vincent ML. Correlates and consequences of early initiation of sexual intercourse. J Sch Health. 1994;64(9):372-7. https://doi.org/10.1111/j.1746-1561.1994.tb06208.x.

5. Halpern CT, Spriggs AL, Martin SL, Kupper LL. Patterns of intimate partner violence victimization from adolescence to young adulthood in a nationally representative sample. J Adolesc Health. 2009;45(5):508-16. https://doi.org/1 0.1016/j.jadohealth.2009.03.011.

6. Santelli JS, Brener ND, Lowry R, Bhatt A, Zabin LS. Multiple Sexual Partners Among U.S. Adolescents and Young Adults. Fam Plan Perspect. 1998:30:271. https://doi.org/10.2307/2991502.

7. Tapert SF, Aarons GA, Sedlar GR, Brown SA. Adolescent substance use and sexual risk-taking behavior. J Adolesc Health. 2001;28(3):181-9. https://doi. org/10.1016/S1054-139X(00)00169-5.

8. Baskin-Sommers A, Sommers I. The co-occurrence of substance use and high-risk behaviors. J Adolesc Health. 2006;38(5):609-11. https://doi.org/10.1 016/j.jadohealth.2005.07.010.

9. Luciana M, Feldstein Ewing SW. Introduction to the special issue: substance use and the adolescent brain: developmental impacts, interventions, and longitudinal outcomes. Dev Cogn Neurosci. 2015;16:1-4. https://doi.org/10.1 016/j.dcn.2015.10.005.

10. Rotz D, Goesling B, Inanc H, Chojnacki G. Economic benefits of delayed sexual activity. Washington, DC: Office of Planning, Research and Evaluation, Administration for Children and Families, U.S. Department of Health and Human Services; 2021

11. Schantz K. Substance use and sexual risk taking in adolescence risk taking in adolescence adolescence: a time for taking chances. 2012. www.a ctforyouth.net. Accessed 15 Jan 2021.

12. Cavazos-Rehg PA, Krauss MJ, Spitznagel EL, Schootman M, Cottler LB, Bierut $\sqcup$. Brief report: pregnant by age 15 years and substance use initiation among US adolescent girls. J Adolesc. 2012;35(5):1393-7. https://doi.org/1 0.1016/j.adolescence.2012.03.001.

13. Gau SSF, Chong M-Y, Yang P, Yen C-F, Liang K-Y, Cheng ATA. Psychiatric and psychosocial predictors of substance use disorders among adolescents. Br J Psychiatry. 2007;190(1):42-8. https://doi.org/10.1192/bjp.bp.106.022871.

14. Ryan SM, Jorm AF, Lubman DI. Parenting factors associated with reduced adolescent alcohol use: a systematic review of longitudinal studies. Aust N Z J Psychiatry. 2010;44(9):774-83. https://doi.org/10.1080/00048674.2010.501759.

15. Shrier LA, Harris SK, Beardslee WR. Temporal associations between depressive symptoms and self-reported sexually transmitted disease among adolescents. Arch Pediatr Adolesc Med. 2002;156(6):599-606. https://doi. org/10.1001/archpedi.156.6.599.
16. Crosby RA, DiClemente RJ, Wingood GM, Sionéan C, Cobb BK, Harrington K. Correlates of unprotected vaginal sex among African American female adolescents. Arch Pediatr Adolesc Med. 2000;154(9):893-9. https://doi.org/1 0.1001/archpedi.154.9.893.

17. Gardner LH, Frank D, Amankwaa LI. A comparison of sexual behavior and self-esteem in young adult females with positive and negative tests for sexually transmitted diseases. ABNF J. 1998;9(4):89-94.

18. Abbott $P$, Chase DM. Culture and substance Abuse: impact of culture affects approach to treatment. Psychiatr Times. 2008;25 https://www.psychia trictimes.com/view/culture-and-substance-abuse-impact-culture-affects-a pproach-treatment. Accessed 24 Mar 2021.

19. Griese. Identfying Sexual Health Protective Factors among Northern Plains AMerican Indian Youth: An Ecological Approach Utilizing Multiple Perspectives. Am Indian Alaska Nativ Ment Heal Res. 2016;23:16-43. https:// doi.org/10.5820/aian.2304.2016.16.

20. Myers T, Bullock SL, Calzavara LM, Cockerill R, Marshall WW, George-Mandoka C. Culture and sexual practices in response to HIV among Aboriginal people living on-reserve in Ontario. Cult Health Sex. 1999;1(1):19-37. https://doi. org/10.1080/136910599301148.

21. Henson M, Sabo S, Trujillo A, Teufel-Shone N. Identifying protective factors to promote health in American Indian and Alaska native adolescents: a literature review. J Prim Prev. 2017;38(1-2):5-26. https://doi.org/10.1007/s10935-016-0455-2.

22. Van Ryzin MJ, Fosco GM, Dishion TJ. Family and peer predictors of substance use from early adolescence to early adulthood: an 11-year prospective analysis. Addict Behav. 2012;37(12):1314-24. https://doi.org/10.1 016/j.addbeh.2012.06.020.

23. Tingey L, Cwik MF, Rosenstock S, Goklish N, Larzelere-Hinton F, Lee A, et al. Risk and protective factors for heavy binge alcohol use among American Indian adolescents utilizing emergency health services. Am J Drug Alcohol Abuse. 2016:42(6):715-25. https://doi.org/10.1080/00952990.2016.1181762.

24. Markham CM, Lormand D, Gloppen KM, Peskin MF, Flores B, Low B, et al. Connectedness as a predictor of sexual and reproductive health outcomes for youth. J Adolesc Health. 2010;46(3):S23-41. https://doi.org/10.1016/j.ja dohealth.2009.11.214.

25. Hadley W, Brown LK, Lescano CM, Kell H, Spalding K, Diclemente R, et al. Parent-adolescent sexual communication: associations of condom use with condom discussions. AIDS Behav. 2009;13(5):997-1004. https://doi.org/10.1 007/s10461-008-9468-z.

26. Chambers R, Tingey L, Mullany B, Parker S, Lee A, Barlow A. Exploring sexual risk taking among American Indian adolescents through protection motivation theory. AIDS Care. 2016;28(9):1089-96. https://doi.org/10.1080/ 09540121.2016 .1164289

27. CDC. Youth Risk Behavior Surveillance System Data. 2019. www.cdc.gov/ yrbs. Accessed 15 Jan 2021.

28. CDC. Youth Risk Behavior Surveillance System Data. 2017. www.cdc.gov/ yrbs. Accessed 7 Oct 2020

29. NCHHSTP, CDC. Health Disparities: American Indians and Alaska Natives. 2020. https://www.cdc.gov/nchhstp/healthdisparities/Americanlndians.html. Accessed 24 Mar 2021

30. Martin JA, Hamilton BE, Osterman MJK, Driscoll AK, Drake P. Births: final data for 2017. Natl Vital Stat Rep. 2018;67:1-8 https://www.cdc.gov/nchs/data_a ccess/Vitalstatsonline.htm. Accessed 28 Dec 2020.

31. CDC. Social Determinants and Eliminating Disparities in Teen Pregnancy. 2019. https://www.cdc.gov/teenpregnancy/about/social-determinants-dispa rities-teen-pregnancy.htm. Accessed 24 Mar 2021.

32. Substance Abuse and Mental Health Services Administration. 2018 NSDUH Detailed Tables 2019. https://www.samhsa.gov/data/report/2018-nsduh-deta iled-tables. Accessed 13 Jan 2021.

33. Chambers R, Patel H, Richards J, Begay J, Littlepage S, Begay M, et al. Feasibility, acceptability, preliminary Impact of Asdzáán Be'eená: an intergenerational, strength-based and culturally grounded program to improve the health of Navajo families. Fam Community (In press). 2021.

34. Shonkoff JP, Boyce WT, McEwen BS. Neuroscience, molecular biology, and the childhood roots of health disparities. JAMA. 2009:301(21):2252-9. https://doi.org/10.1001/jama.2009.754

35. StateUniversity.com. Stages of Growth Child Development - Early Childhood (Birth to Eight Years), Middle Childhood (Eight to Twelve Years). Education Encyclopedia. https://education.stateuniversity.com/pages/1826/ChildDevelopment-Stages-Growth.html\#ixzz0j0jMHgRB. Accessed 24 Mar 2021.

36. National Research Council (US) Panel to Review the Status of Basic Research on School-Age Children. Conclusion: The Status of Basic Research on 
Middle Childhood. In: Collins WA, editor. Development During Middle Childhood: The Years From Six to Twelve. Washington, DC: The National Academies Press; 1984. doi:10.17226/56.

37. Halfon N, Larson K, Lu M, Tullis E, Russ S. Lifecourse health development: past, present and future. Matern Child Health J. 2014;18(2):344-65. https:// doi.org/10.1007/s10995-013-1346-2.

38. Light H, Martin R. American Indian families. J Am Indian Educ. 1986;26:1-5.

39. Tsethlikai M, Murray DW, Meyer AM, Sparrow J. Reflections on the relevance of "self-regulation" for native communities (OPRE brief \#2018-64). Washington, DC: Office of Planning, Research and Evaluation, Administration for Children and Families, U.S. Department of Health and Human Services; 2018. https://fpg.unc.edu/publications/reflections-relevance-self-regulationnative-communities. Accessed 24 Mar 2021

40. Bahar S, editor. Health Behavior: Emerging Research Perspectives. 1st ed: Springer US; 1998

41. Wrotniak BH, Epstein LH, Paluch RA, Roemmich JN. Parent weight change as a predictor of child weight change in family-based behavioral obesity treatment. Arch Pediatr Adolesc Med. 2004;158(4):342-7. https://doi.org/10.1 001/archpedi.158.4.342

42. Craig Rushing S, Stephens D, Shegog R, Torres J, Gorman G, Jessen C, et al. Healthy native youth: improving access to effective, Culturally-Relevant Sexual Health Curricula. Front Public Health. 2018;6:225. https://doi.org/10.33 89/fpubh.2018.00225.

43. Brackney DE, Cutshall M. Prevention of type 2 diabetes among youth: a systematic review, implications for the school nurse. J Sch Nurs. 2014;31(1): 6-21. https://doi.org/10.1177/1059840514535445.

44. American Diabetes Association. 12. Children and Adolescents: Standards of Medical Care in Diabetes_-2018. Diabetes Care. 2018;41(Supplement 1): S126-36. https://doi.org/10.2337/dc18-S012.

45. Jackson C, Henriksen L, Foshee VA. The authoritative parenting index: predicting health risk behaviors among children and adolescents. Health Educ Behav. 1998;25(3):319-37. https://doi.org/10.1177/1090198198025003 07.

46. Silverberg S, Small S. Parental monitoring, family structure and adolescent substance use; 1991.

47. Gregory T, Engelhardt D, Lewkowicz A, Luddy S, Guhn M, Gadermann A et al. Validity of the middle years development instrument for population monitoring of student wellbeing in Australian school children. Child Indic Res. 2019;12(3):873-99. https://doi.org/10.1007/s12187-018-9562-3.

48. Oman RF, Vesely SK, Mcleroy KR, Harris-Wyatt V, Aspy CB, Rodine S, et al. Reliability and validity of the youth asset survey (YAS). J Adolesc Health. 2002;31(3):247-55. https://doi.org/10.1016/S1054-139X(02)00363-4.

49. Sexual Risk Avoidance Education Program. Middle School Participant Entry Survey. https://www.sraepas.com/tta-resources/. Accessed 15 Apr 2021.

50. Leach CW, van Zomeren M, Zebel S, Vliek ML, Pennekamp SF, Doosje B, et al. Group-level self-definition and self-investment: a hierarchical (multicomponent) model of in-group identification. J Pers Soc Psychol. 2008;95(1):144-65. https://doi.org/10.1037/0022-3514.95.1.144.

51. Moos RH, Moos BS. A typology of family social environments. Fam Process. 1976;15(4):357-71. https://doi.org/10.1111/j.1545-5300.1976.00357.x.

52. Bogenschneider K, Small SA, Tsay JC. Child, parent, and contextual influences on perceived parenting competence among parents of adolescents. J Marriage Fam. 1997;59(2):345-62. https://doi.org/10.2307/353475.

53. Jaccard J, Dittus PJ, Gordon W. Maternal correlates of adolescent sexual and contraceptive behavior. Fam Plan Perspect. 1996;28(4):159-85. https://doi. org/10.2307/2136192.

54. Humeniuk R, Henry-Edwards S, Ali R, Poznyak V, Monteiro M. The alcohol, smoking and substance involvement screening test (ASSIST): manual for use in primary care; 2010.

55. Campis LK, Lyman RD, Prentice-Dunn S. The parental locus of control scale: development and validation. J Clin Child Psychol. 1986;15(3):260-7. https:// doi.org/10.1207/s15374424jccp1503_10

56. Dumka LE, Stoerzinger HD, Jackson KM, Roosa MW. Examination of the cross-cultural and cross-language equivalence of the parenting self-agency measure. Fam Relat. 1996;45(2):216-22. https://doi.org/10.2307/585293.

57. Fok CCT, Allen J, Henry D, Mohatt GV. Multicultural mastery scale for youth: multidimensional assessment of culturally mediated coping strategies. Psychol Assess. 2012;24(2):313-27. https://doi.org/10.1037/a0025505.

58. Sexual Risk Avoidance Education Program. Middle School Participant Exit Survey. https://www.sraepas.com/wp-content/uploads/2020/07/MiddleSchool-Participant-Exit-Survey.pdf. Accessed 15 Apr 2021.
59. Abuse S, Administration MHS. Results from the 2009 National Survey on Drug Use and Health: Volume I. In: Summary of National Findings. Off Appl Stud NSDUH Ser H-38A, HHS Publ No SMA 10-4586Findings; 2010. http:// www.oas.samhsa.gov. Accessed 15 Jan 2021.

60. de Ravello L, Everett Jones S, Tulloch S, Taylor M, Doshi S. Substance use and sexual risk behaviors among American Indian and Alaska native high school students. J Sch Health. 2014;84(1):25-32. https://doi.org/10.1111/ josh.12114.

61. Westling E, Andrews JA, Hampson SE, Peterson M. Pubertal timing and substance use: the effects of gender, parental monitoring and deviant peers. J Adolesc Health. 2008;42(6):555-63. https://doi.org/10.1016/j.jadohea Ith.2007.11.002.

62. Whitbeck $L B$, Armenta BE. Patterns of substance use initiation among indigenous adolescents. Addict Behav. 2015;45:172-9. https://doi.org/10.101 6/j.addbeh.2015.01.006.

63. Schinke SP, Cole KCA, Fang L. Gender-specific intervention to reduce underage drinking among early adolescent girls: A test of a computermediated, mother-daughter program. J Stud Alcohol Drugs. 2009;70:70-7. https://doi.org/10.15288/jsad.2009.70.70.

64. Rayle AD, Kulis S, Okamoto SK, Tann SS, LeCroy CW, Dustman P, et al. Who is offering and how often? Gender differences in drug offers among American Indian adolescents of the southwest. J Early Adolesc. 2006;26(3): 296-317. https://doi.org/10.1177/0272431606288551.

65. Velleman RDB, Templeton $L$, Copello AG. The role of the family in preventing and intervening with substance use and misuse: a comprehensive review of family interventions, with a focus on young people. Drug Alcohol Rev. 2005;24(2):93-109. https://doi.org/10.1080/ 09595230500167478

66. Widman L, Evans R, Javidi H, Choukas-Bradley S. Assessment of parentbased interventions for adolescent sexual health: a systematic review and meta-analysis. JAMA Pediatr. 2019;173(9):866-77. https://doi.org/10.1001/ja mapediatrics.2019.2324.

67. Abuse S, Administration MHS. Office of the Surgeon General. Prevention programs and policies. In: facing addiction in America: the surgeon General's report on alcohol, drugs, and health [internet]. Washington (DC): U.S. Department of Health \& Human Services; 2016. https://www.ncbi.nlm. nih.gov/books/NBK424850/

68. Guilamo-Ramos V, Jaccard J, Dittus P, Bouris A, Gonzalez B, Casillas E, et al. A comparative study of interventions for delaying the initiation of sexual intercourse among Latino and black youth. Perspect Sex Reprod Health. 2011:43(4):247-54. https://doi.org/10.1363/4324711.

69. Wyckoff SC, Miller KS, Forehand R, Bau JJ, Fasula A, Long N, et al. Patterns of sexuality communication between preadolescents and their mothers and fathers. J Child Fam Stud. 2008;17(5):649-62. https://doi.org/10.1007/s10826007-9179-5.

70. Salazar MK. Interviewer Bias: how it affects survey research. AAOHN J. 1990; 38(12):567-72. https://doi.org/10.1177/216507999003801203.

71. Markham CM, Craig Rushing S, Jessen C, Lane TL, Gorman G, Gaston A, et al. Factors associated with early sexual experience among American Indian and Alaska native youth. J Adolesc Health. 2015:57(3):334-41. https://doi. org/10.1016/j.jadohealth.2015.06.003.

\section{Publisher's Note}

Springer Nature remains neutral with regard to jurisdictional claims in published maps and institutional affiliations.

Ready to submit your research? Choose BMC and benefit from:

- fast, convenient online submission

- thorough peer review by experienced researchers in your field

- rapid publication on acceptance

- support for research data, including large and complex data types

- gold Open Access which fosters wider collaboration and increased citations

- maximum visibility for your research: over $100 \mathrm{M}$ website views per year

At BMC, research is always in progress.

Learn more biomedcentral.com/submissions 